\title{
A sensitive survey for water masers towards Bok globules
}

\author{
I. de Gregorio-Monsalvo' ${ }^{1}$, J. F. Gómez ${ }^{2}$, O. Suárez ${ }^{3}$, T. B. H.

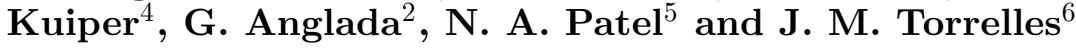 \\ ${ }^{1}$ European Southern Observatory, Alonso de Córdova 3107, Vitacura, Santiago, Chile , \\ email: idegrego@eso.org. \\ ${ }^{2}$ Instituto de Astrofísica de Andalucía (CSIC), Apartado 3004, E-18080 Granada, Spain. \\ ${ }^{3}$ Laboratorio de Astrofísica Espacial y Física Fundamental (INTA), Apartado 50727, E-28080 \\ Madrid, Spain. \\ ${ }^{4}$ Jet Propulsion Laboratory, California Institute of Technology, 4800 Oak Grove Drive, \\ Pasadena, CA 91109, USA. \\ ${ }^{5}$ Harvard-Smithsonian Center for Astrophysics, 60 Garden Street, Cambridge, MA 02138, \\ USA. \\ ${ }^{6}$ Instituto de Ciencias del Espacio (CSIC) and Institut d'Estudis Espacials de Catalunya, \\ Facultat de Física, Planta 7, Universitat de Barcelona, Av. Diagonal 647, E-08028 Barcelona, \\ Spain
}

\begin{abstract}
In this work we report the most sensitive water maser survey towards Bok globules to date, using NASA's $70 \mathrm{~m}$ antenna in Robledo de Chavela (Spain). We observed 207 positions within the Clemens \& Barvainis catalog that show indications of possible star formation or with a high probability of harboring a young stellar object. With this survey we have increased the number of Bok globules known to present water maser emission from three to nine. We have complemented these results with interferometric high-angular resolution observations towards some of our detections.
\end{abstract}

Keywords. ISM: globules, ISM:molecules, masers, stars:formation, surveys.

\section{Motivation}

Bok globules, which are the smallest and more isolated molecular clouds in our galaxy, are important laboratories for the study of star formation processes and protostar evolution with a lower chance of contamination from multiple generations of young stellar objects (YSOs) within the same region. They may span a wide range of evolutionary stages, and low- and intermediate-mass star formation can take place inside these clouds (Bok \& Reilly 1947, Yun \& Clemens 1992).

Water masers are powerful observational tools for characterizing the age of low-mass protostars (most of them are associated with Class 0 protostars) and the physical conditions of the gas surrounding YSOs (Moscadelli et al. 2006). In addition, these masers tend to be located very close (a few hundred AUs) to their powering sources in low-mass YSOs (Claussen et al. 1998) and this makes them well-suited to locating the central exciting sources of mass-loss activity in low-mass star forming regions.

Since Bok globules are associated with YSOs in different evolutionary stages and there are several evolutionary aspects related to water maser emission, we have searched for water masers at $22 \mathrm{GHz}$ towards these clouds using both single-dish and interferometric techniques. 


\section{Single-dish survey for water masers towards Bok globules}

We have used the NASA's 70 m antenna in Robledo de Chavela, near Madrid, Spain, to search for water maser emission at $22 \mathrm{GHz}$ towards 207 positions that include radio continuum sources, centers of molecular outflows, peaks of high-density molecular tracers, and IRAS sources, i.e., targets that may indicate the presence of star formation activity.

As a result, we obtained seven detections (six of them are new) towards CB 3, CB 34, CB 54, CB 65, CB 101, CB 199, and CB 232, increasing from three known sources (CB 3, CB 39, and CB 205) to nine the number of known Bok globules in the Clemens \& Barvainis (1988) catalog associated with this kind of emission (Gómez et al. 2006).

\section{High-angular resolution observations}

As a complement to our single-dish survey we have performed interferometric observations using the Very Large Array (VLA) towards some of our water maser detections and processed archive data in the case of CB 3. We have used these interferometric observations to study the physical conditions and kinematics of the innermost areas around the central YSOs, as well as to determine accurate positions for the masers and additional information about their powering sources.

We have detected water maser emission with the VLA in CB 3, CB 54, CB 101, and CB 232. All of these masers are associated with regions that show bipolar molecular outflows and young multiple stellar systems in different stages of evolution, with the exception of CB 101 which we propose to be associated with an evolved object, possibly a Mira star (see de Gregorio-Monsalvo et al. 2006).

Thanks to the accurate positions provided by the interferometric observations, we obtained information about the powering sources in CB 3 (where masers are located close to the Class 0 object CB 3-mm), CB 54 (where maser emission is associated to a near-IR elongated feature named CB 54 YC1-SW), and CB 232 (with the water maser emission located at the position of the near-IR source CB 232 YC1-I). We have confirmed that $\mathrm{CB} 54 \mathrm{YC} 1-\mathrm{SW}$ is the powering source of the maser emission and possibly of the mass loss phenomenon in CB 54, with recent deep VLA-BnA observations that show a faint radio continuum source at that position (de Gregorio-Monsalvo et al. in preparation).

\section{Acknowledgements}

GA, JFG, and JMT acknowledge partial financial support from grant AYA2005-08523C03 of the Spanish MEC (co-funded with FEDER funds). GA, IdG, JFG, and JMT acknowledge support from Junta de Andalucía grant FQM-1747. GA and JFG are also partially supported by Junta de Andalucía grant TIC-126.

\section{References}

Bok, B. J., \& Reilly, E. F. 1947, ApJ, 105, 255

Claussen, M. J., Marvel, K. B., Wootten, A., \& Wilking, B. A. 1998, ApJ, 507, L79

Clemens, D. P. \& Barvainis, R. 1988, ApJS, 68, 257

de Gregorio-Monsalvo, I., Gómez, J. F., Suárez, O., Kuiper, T. B. H., Anglada, G., Patel, N. A., \& Torrelles, J. M. 2006, AJ, 132, 2584

Gómez, J. F., de Gregorio-Monsalvo, I., Suárez, O, \& Kuiper,T. B. H. 2006, AJ, 132, 1322

Moscadelli, L., Testi, L., Furuya, R. S., Goddi, C., Claussen, M., Kitamura, Y., \& Wootten, A. 2006, A\&A, 446, 985

Yun, J. L., \& Clemens, D. P. 1992, ApJ, 385, L21 